\title{
A Novel Method for In Vivo Imaging of Solitary Lung Nodules Using Navigational Bronchoscopy and Confocal Laser Microendoscopy
}

\author{
T. Hassan ${ }^{1,2,3} \cdot$ N. Piton ${ }^{4}$ - S. Lachkar ${ }^{1,2,3} \cdot$ M. Salaün ${ }^{1,2,3} \cdot$ L. Thiberville ${ }^{1,2,3}$
}

Received: 16 April 2015 / Accepted: 19 July 2015/Published online: 28 July 2015

(c) The Author(s) 2015. This article is published with open access at Springerlink.com

\begin{abstract}
Solitary pulmonary nodules (SPN) have become increasingly prevalent and diagnostic management remains challenging. We demonstrate a novel technique in which probe-based confocal endomicroscopy (pCLE) could be performed to microimage SPN in vivo and in realtime. Two confocal wavelengths (488 and $660 \mathrm{~nm}$ with methylene blue (MB)) were used for elastin network and cellular imaging, respectively using pCLE in conjunction with r-EBUS and virtual navigation. In the first case, the 1-mm Alveoflex ${ }^{\circledR}$ was used to image a metastatic melanoma in a subcentimetric nodule in the right middle lobe. In the next case, a malignant $2-\mathrm{cm}$ nodule in the posterior segment of the upper lobe was imaged using the smaller 0.6-mm Cholangioflex ${ }^{\circledR}$. Lastly, we present a benign case revealing confocal characteristics of a nodular lipid pneumonitis. This reports for the first time the feasibility and utility of pCLE in vivo microimaging of SPN using either the Alveoflex or Cholangioflex miniprobes in addition to $660 \mathrm{~nm} / \mathrm{MB}$ imaging.
\end{abstract}

Electronic supplementary material The online version of this article (doi:10.1007/s00408-015-9769-8) contains supplementary material, which is available to authorized users.

L. Thiberville

luc.thiberville@chu-rouen.fr

1 Clinique Pneumologique, Rouen University Hospital, 1 rue de Germont, 76031 Rouen Cedex, France

2 EA 4108, QuantIF, Rouen University, Rouen, France

3 CIC-INSERM 1404, Rouen University Hospital, Rouen Cedex, France

4 Pathology Department, Rouen University Hospital, Rouen, France
Keywords Solitary pulmonary nodules $\cdot$ Probe-based confocal microendoscopy $\cdot$ Lung malignancy .

Bronchoscopy

$\begin{array}{ll}\text { Abbreviations } \\ \text { CT } & \text { Computed tomography } \\ \text { EGFR } & \text { Epidermal growth factor receptor } \\ \text { EMN } & \text { Electromagnetic navigation bronchoscopy } \\ \text { MB } & \text { Methylene blue } \\ \text { pCLE } & \text { Probe-based confocal laser endomicroscopy } \\ \text { PET } & \text { Postive emission tomography } \\ \text { r-EBUS } & \text { Radial probe ultrasound endobronchoscopy } \\ \text { SPN } & \text { Solitary pulmonary nodule }\end{array}$

\section{Introduction}

Solitary pulmonary nodules (SPN) have become more prevalent and due to the increasing reports of peripheral lung adenocarcinoma, the management of SPNs is becoming more challenging [1-3]. New endoscopic techniques such as navigation bronchoscopy have improved diagnostic yield [4-6].

Probe-based confocal laser endomicroscopy (pCLE) is a new technique that can microscopically image the alveoli in vivo during standard flexible bronchoscopy [7-9]. A thin and flexible miniprobe called Alveoflex ${ }^{\circledR}$ (Mauna Kea Technologies, Paris, France) is advanced through the working channel of the flexible bronchoscope to reach the distal lung. Thinner and more flexible probes are available for bile duct exploration $\left(\right.$ Cholangioflex $\left.{ }^{\circledR}\right)$ that may be useful for subsegmental bronchi that are more distal and difficult to access.

Two different wavelengths are available: $488 \mathrm{~nm}$ used for autofluorescence microimaging of the elastin network 
imaging, while the $660 \mathrm{~nm}$ with topical application of methylene blue (MB) can be used for cellular imaging [10]. This technique could potentially allow improved targeting of the biopsy area, with in vivo cellular and microstructural imaging of SPNs.

To this day, there are currently no reports published on the utility of pCLE for the diagnosis of SPN. We present here three cases showing how the r-EBUS/navigational technique can be enhanced with pCLE.

\section{Method}

To image SPNs in vivo and in real-time, we developed a technique based on pCLE of the distal lung (Alveoscopy) $[9,10]$. The method used r-EBUS coupled with an external sheath and virtual navigation, in which the confocal miniprobe was then introduced into the sheath in order to image the target before sampling.

The endobronchial path to the target was first determined using a navigational software (superDimension ${ }^{\mathrm{TM}}$ ). The bronchus of interest was aligned in three dimensions toward the SPN. At this point, the distance between the closest subsegmental bronchi visualized and the SPN of interest was measured. During bronchoscopy under local anesthesia, we applied the technique previously described for the evaluation of SPN using r-EBUS of $20 \mathrm{MHz}$ (Olympus UM-3R, Tokyo, Japan), 1.4-mm radial EBUS probe (UM-S20-17S, Olympus, Tokyo, Japan) and a 1.9-mm external sheath (K-201 Olympus, Tokyo, Japan) introduced into the 2-mm working channel of a 4-mm flexible bronchoscope (MP60 model; Olympus, Tokyo, Japan) [11].

A stopper was positioned on the confocal miniprobe to ascertain that the tip of the confocal miniprobe coincides with the tip of the external guide sheath (Fig. 1a). Once the nodule was located with r-EBUS, the confocal miniprobe was introduced through the same guide sheath. To image the alveoli, the bronchial wall was penetrated. A solid pattern was identified as areas in which normal alveolar network was not recognizable and associated with a dense appearance.

Subsequent in vivo cellular imaging was obtained at $660 \mathrm{~nm}$ after topical administration of $10 \mu \mathrm{l}$ of $1 \%$ methylene blue (Proveblue ${ }^{\circledR}$, Marseille, France) using a 1.4-mm cytology brush (BC-204D-2010, Olympus, Tokyo Japan). This was followed by biopsies (FB-233D, Olympus, Tokyo, Japan).

Due to the probe stiffness, the 1-mm Alveoflex ${ }^{\circledR}$ miniprobe was used to evaluate SPNs in the lingular, middle, and lower lobes in addition to the anterior segments of the upper lobes $[9,10]$. Meanwhile, the 0.6-mm Cholangioflex ${ }^{\circledR}$ was used to image the apical and posterior segments of the upper lobes. Recorded image analysis was performed using the Medviewer ${ }^{\circledR} 1.1$ software (Mauna Kea, Paris, France,) and compared with histopathology.
The subjects discussed in this study were recruited as part of the NOVIDEM trial (ClinicalTrials.gov identifier: NCT01931579) and approved by the ethical committee of the University Hospital, Rouen to include both Alveoflex ${ }^{\circledR}$ and Cholangioflex ${ }^{\circledR}$ probes. All patients gave informed consent. The procedures were performed by one endoscopist (LT) under local anesthesia.

\section{Results}

\section{Case 1:9-mm Right Middle Lobe Nodule Using the 1-mm Alveoflex ${ }^{\circledR}$}

The first example involves a 74-year-old male who was surgically treated for both malignant melanoma of the left forearm and a squamous cell carcinoma of the skin 2 years previously. A follow-up CT scan revealed a 9-mm diameter nodule in the right middle lobe. With superDimension ${ }^{\mathrm{TM}}$, we planned the road map of the subsegmental bronchi distal to RB4, pre-empting that the SPN is $2 \mathrm{~cm}$ away from the last attainable bronchus (Fig. 1b).

Once the nodule was located using r-EBUS, confocal imaging at $488 \mathrm{~nm}$ was performed at this level (Fig. 1c). This revealed a compact, destroyed alveoli with thickened fibers (Fig. 1d). The MB/660-nm confocal analysis revealed increased cellularity of regular, small $(<20 \mu \mathrm{m})$ individual cells (Fig. 1e). There were areas of decreased fluorescence signal suggesting melanin production which has been shown to absorb light at $660 \mathrm{~nm}$ [12]. Due to the high level of suspicion for malignancy in this history and abnormal pCLE findings, a fiducial marker was placed into the nodule during the same procedure.

The biopsies obtained through the guide sheath during the endoscopy revealed small malignant cells composing of eosinophilic cytoplasm containing large nuclei, expressing reliable melanoma tumor markers. The molecular analysis of the biopsy sample showed BRAF-V600E mutation (Fig. 1f). These findings were consistent with a lung metastasis of melanoma, which was later treated with Cyberknife $^{\circledR}$.

\section{Case 2: A 2-cm Nodule in the Right Upper Lobe Using the 0.6-mm Cholangioflex ${ }^{\circledR}$}

The case involves a 51-year-old male smoker with a history of supracricoid laryngectomy for squamous cell carcinoma 3 years previously. He presented with left-sided hemiparesis, and a subsequent full-body CT scan revealed multiple intracranial lesions in addition to a right lung apical mass (Fig. 2a). Using virtual navigation, we planned the road map distal to the right upper lobe bronchus, anticipating a difficult course involving the posterior and 

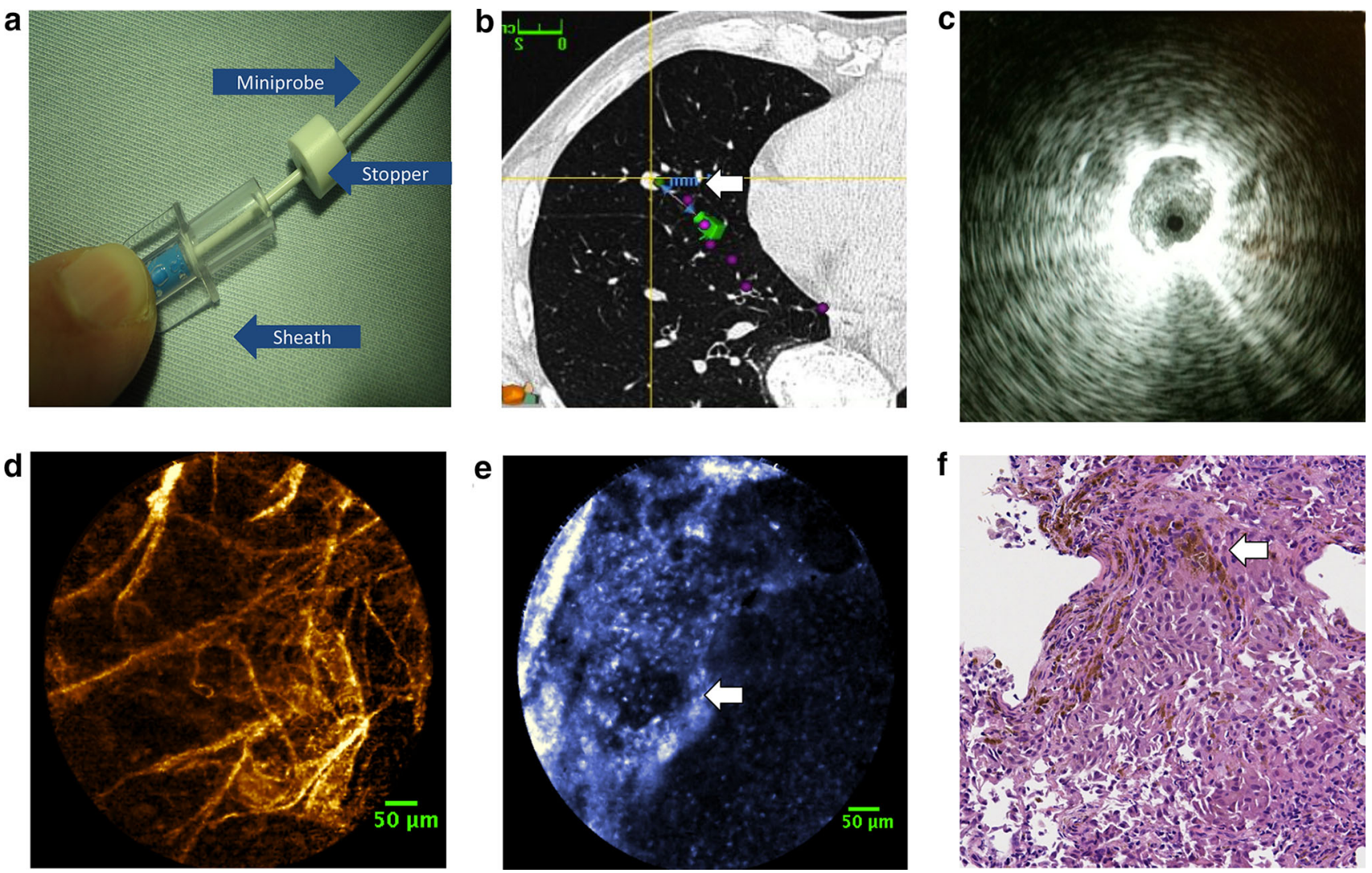

Fig. 1 a A stopper is used to determine that the tip of the confocal miniprobe coincides with the tip of the external guide sheath which was firstly used by r-EBUS to locate the nodule. $\mathbf{b}$ The layout of the virtual bronchoscopy using the superDimension ${ }^{\mathrm{TM}}$ software to 3-dimensionally identify the pathway to the 9-mm nodule (white arrow) in the right middle lobe (only one dimension demonstrated here). $\mathbf{c}$ The r-EBUS image of the right middle lobe revealing a small hypoechoic area in RB4. d Right middle lobe nodule alveoscopic image at $488 \mathrm{~nm}$ showing distorted, irregular elastic fiber network with an area that appeared more compact. Some alveoli remain

then, the medial subsegment of RB1 (Fig. 2b). The 2-cm nodule was measured to be $3 \mathrm{~cm}$ away from the last attainable bronchus.

The Cholangioflex ${ }^{\circledR}$ was introduced into the guide sheath once r-EBUS revealed the nodule of interest (Fig. 2c). The microimaging revealed a dense appearance of solid tissue with unrecognizable elastin network (Fig. 2d). A $660 \mathrm{~nm} / \mathrm{MB}$ imaging revealed scattered large cells of 25-30 microns. Decreased areas of fluorescent signal also suggest local necrosis (Fig. 2e). This coincides with the bronchial biopsy which was reported to be paucicellular, but displayed necrotic materials. In order to secure the diagnosis, the patient subsequently had a wedge resection a week later which revealed large cell undifferentiated carcinoma with extensive necrosis (Fig. 2f). recognizable however, are distorted (scale bar $50 \mu \mathrm{m}$ using the Alveoflex ${ }^{\circledR}$ ). e Alveoscopic image at $660 \mathrm{~nm}$ with topical MB administration revealing increasing cellularity of regular, small individual cells, and areas of decreased fluorescence that may correspond to melanin disposition (white arrow) (scale bar $50 \mu \mathrm{m}$ using the Alveoflex ${ }^{\circledR}$ ). f Histopathological analysis stained with haematoxylin and eosin revealing melanoma with high nucleocytoplasmic ratios and prominent nucleoli, in addition to melanin production which appears as prominent pigment synthesis (white arrow)

\section{Case 3: Lipid-Lung Disease Using the Alveoflex ${ }^{\circledR}$ and $660 \mathrm{~nm} / \mathrm{MB}$ Cellular Imaging}

A 55-year-old female smoker who presented with shortness of breath admitted to consuming large quantities of liquid paraffin due to her history of chronic constipation. Her chest CT scan revealed nodular consolidation in the left lower and right middle lobe, which displayed areas of negative Hounsfield values.

Again, virtual navigation was used to evaluate the endoscopic path for both lesions in RB5 and LB7 (Fig. 3a, b). The distal and then medial subsegmental bronchus in the right middle lobe appeared stenosed, and indeed were not accessible using r-EBUS. However, using the Alveolflex ${ }^{\circledR}$ Miniprobe for confocal imaging, $100 \mu \mathrm{m}$ fluorescent and 

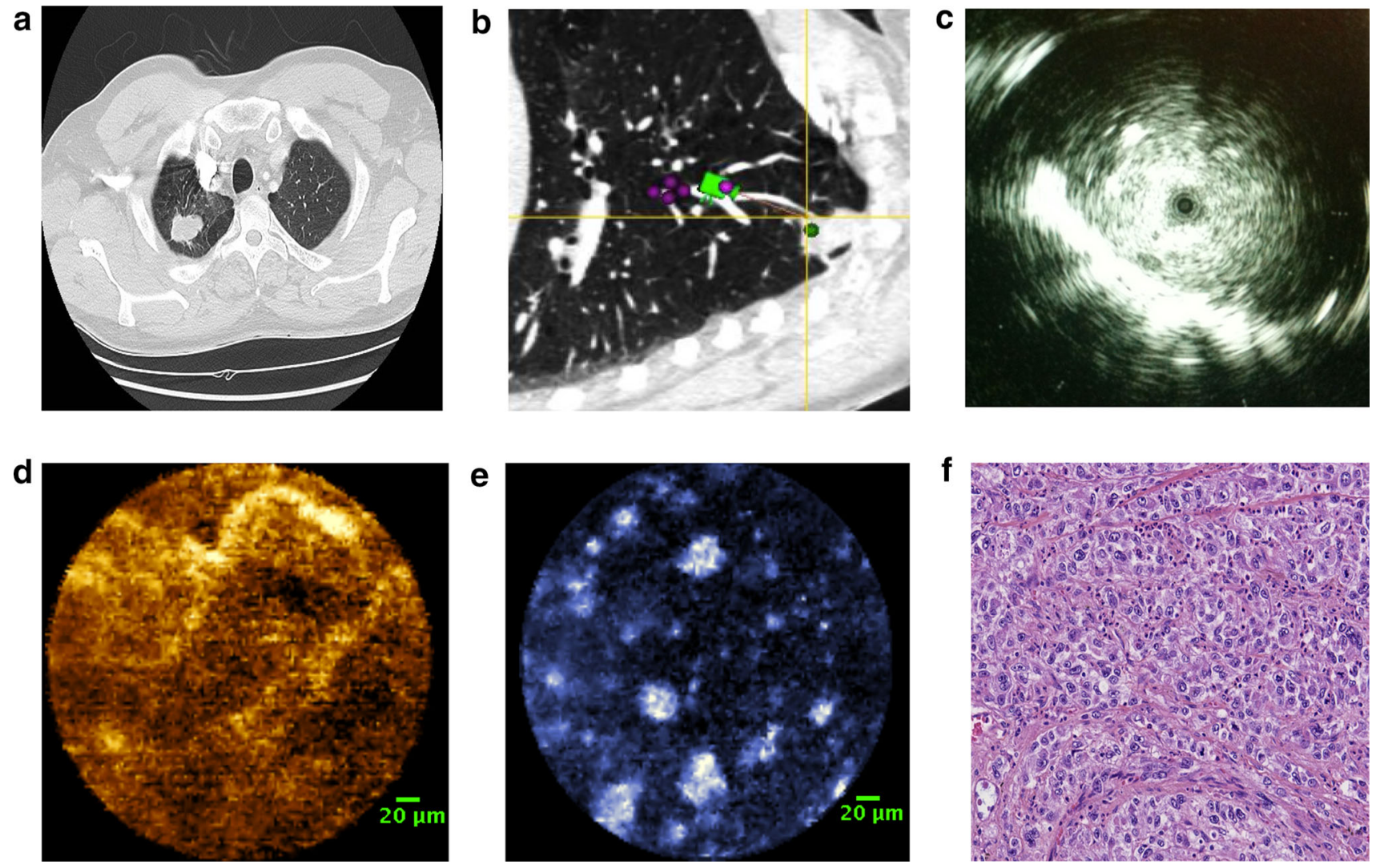

Fig. 2 a Chest CT scan revealing the 2-cm nodule in the right upper lobe. b The superDimension ${ }^{\mathrm{TM}}$ analysis is used to plan the road map to the nodule, pre-empting the site of interest is $3 \mathrm{~cm}$ away from the last attainable pathway in RB1. c The r-EBUS image of the right upper lobe revealing a hypoechoic area in RB1. d Right upper lobe nodule alveoscopic image at $488 \mathrm{~nm}$ showing dense appearance of solid tissue with unrecognizable elastin network (scale bar $20 \mu \mathrm{m}$

mobile round structures were elicited in RB5 that appeared characteristic of lipid droplets (Fig. 3c and online video). The elastin network was distorted and dense. The $660 \mathrm{~nm} /$ MB study revealed paucicellular regions with dark hollow areas (Fig. 3d). Similar findings were observed in LB7. The biopsy reported chronic inflammatory changes in contact with optically empty vacuoles, which confirmed the suspected diagnosis of lipid pneumonitis (Fig. 3e).

\section{Discussion}

This work shows the potential utility of pCLE coupled with $\mathrm{MB} / 660 \mathrm{~nm}$ study in the evaluation of SPNs. The 1-mm Alveoflex ${ }^{\circledR}$ miniprobe was successfully used to assess a subcentimetric nodule, while a $2-\mathrm{cm}$ nodule located in the posterior segment of the upper lobe was evaluated using the 0.6-mm Cholangioflex ${ }^{\circledR}$ miniprobe. In the third case, we present a benign case with a high suspicion for lipidlung disease from the history, with specific characteristics using the Cholangioflex ${ }^{\circledR}$ ). e Alveoscopic image at $660 \mathrm{~nm}$ after topical MB administration revealing scattered large cells with areas of decreased fluorescent signal which may correspond to areas of necrosis (scale bar $20 \mu \mathrm{m}$ using the Cholangioflex ${ }^{\circledR}$ ). f Histopathology analysis stained with haematoxylin and eosin shows a proliferation of undifferentiated large tumor cells

on the confocal imaging. A confocal imaging at $488 \mathrm{~nm}$ of a normal alveoli duct is illustrated in Fig. $3 \mathrm{f}$ for comparison.

The diagnosis of subcentimetric nodules is challenging even with standard guidelines $[3,13,14]$. In the first case, we demonstrated that subcentimetric nodules could be reached with this technique. Due to the abnormal areas identified in the MB/660 nm imaging which raised our suspicion index for malignancy, we decided to place a fiducial marker during the same procedure without a second bronchoscopy.

In the second case, we demonstrated that confocal imaging of a distal, difficult-to-reach nodule is feasible with this combined technique. However, specimens obtained by forceps biopsies using navigation bronchoscopy are often small, and subject to crush artifact, especially in cases of necrotic tumors. In this case, despite localizing the SPN using r-EBUS, the endoscopic biopsies revealed necrosis. However, the MB/660 nm study elicited images of unidentified large scattered cells that were 

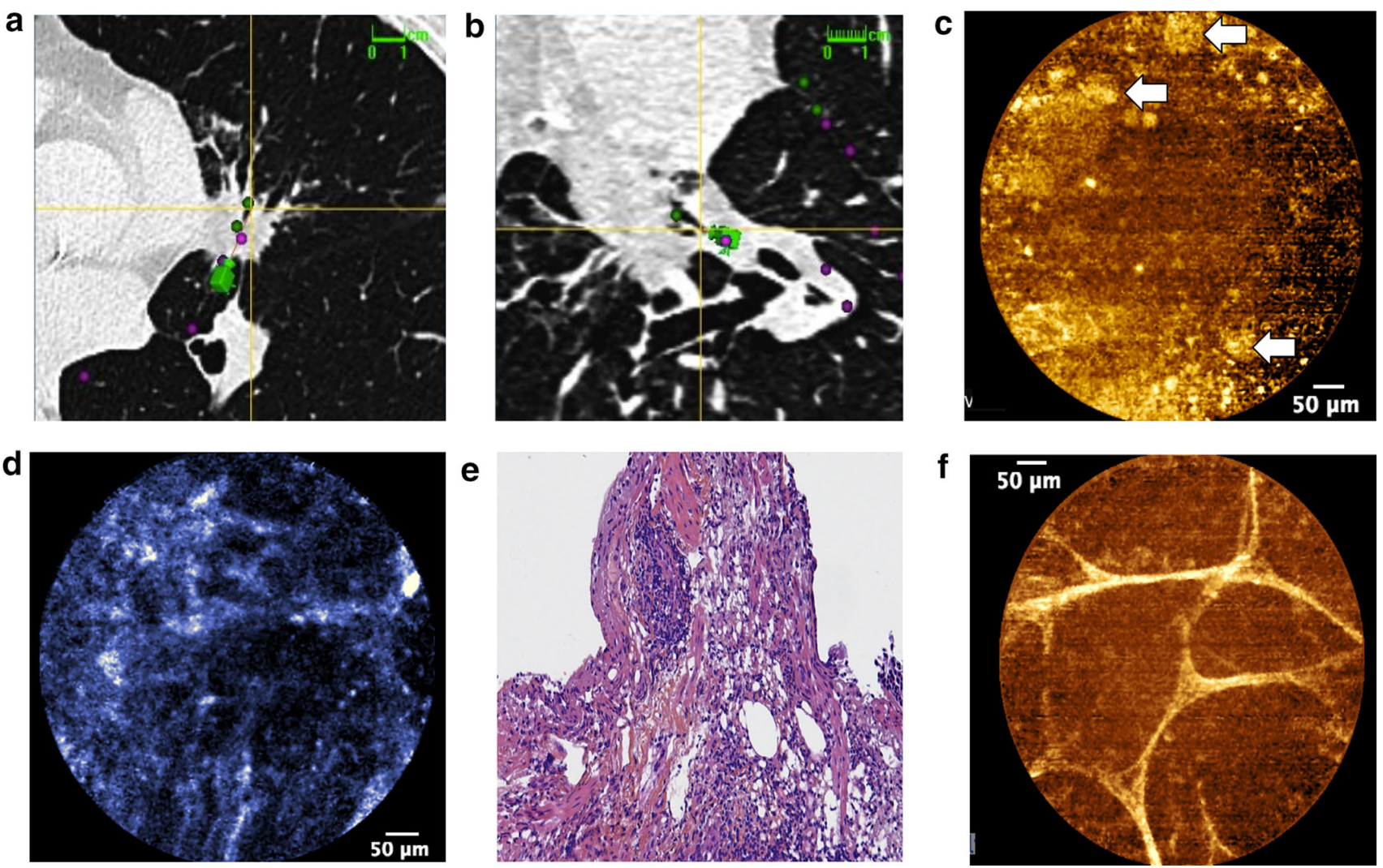

Fig. 3 The superDimension ${ }^{\mathrm{TM}}$ analysis is used to plan the road map to the nodules in a RB5 and b LB7. (The r-EBUS technique did not locate any of the nodule). c Confocal imaging at 488-nm displayed lipid droplets (white arrow) with distorted elastin network (see also the kinetics of the lipid droplets on the online video) (scale bar $50 \mu \mathrm{m}$ using the Alveoflex ${ }^{\circledR}$ ) while d the $660-\mathrm{nm}$ imaging revealed

different from previously described alveolar cells from normal, healthy lungs [9]. Therefore, similar to the first case, this case illustrates the added benefit of MB/660 nm evaluation for in vivo cellular imaging, in addition to the analysis of elastin network using the standard 448-nm excitation. However, the in vivo confocal imaging obtained did not obviate the need for surgical wedge resection to secure the diagnosis. In an unpublished report, we have observed that topical MB/660 nm pCLE makes it possible to image the cellular organization of SPNs in which we identified possible pCLE characteristics of small-cell lung cancer from other histological type [15].

In the third case, it was the pCLE, not r-EBUS that guided the site for the biopsies which displayed histopathology characteristics (empty vacuoles) relevant to those elicited using confocal imaging (lipid droplets). Therefore, we demonstrated that microimaging the SPN in vivo using pCLE could potentially optimize the site for targeted biopsies, perhaps adding diagnostic value to other techniques such as r-EBUS and navigation bronchoscopy. paucicellular areas with dark hollows (white arrow) (scale bar $50 \mu \mathrm{m}$ using the Alveoflex ${ }^{\circledR}$ ). e Histopathology analysis stained with haematoxylin and eosin displayed normal bronchial epithelial cells with lymphocytes infiltration and optically empty vacuoles. f 488-nm confocal imaging of a normal axial elastic backbone of an alveolar duct

The evidence for the potential role of pCLE is growing. In previous works, limitations of pCLE have been defined including artifacts linked to compression of fragile parenchyma structures as well as difficulties in interpretation of elastin network which may vary from different individuals $[9,10]$. However, as the site of evaluation is specific and guided by navigational bronchoscopy, these limitations are appearing less important for SPNs compared to diffuse interstitial diseases. However, a potential limitation for pCLE in the diagnosis of SPNs lies in identifying the difference between malignant and benign nodules which may exhibit similar confocal characteristics.

In conclusion, although it is too early to say if pCLE will be able to replace conventional biopsy for SPN, we show here the utility of pCLE for in vivo microimaging and optimizing target biopsies in three cases of SPNs. Large future prospective studies including comparison with navigational bronchoscopy without confocal imaging and/or CT-guided biopsies are required to predict the presence of malignancy with high accuracy using standardized, reproducible descriptors. 
Acknowledgments We acknowledge the support of the European Respiratory Society, Fellowship STRTF-2014-5440, and of the French Ministry of Health/INCa, programme hospitalier de recherche clinique "Cancer," 2011 (\# PHRC11-192).

\section{Compliance with Ethical Standards}

Conflict of interest LT received a research grant from Mauna Kea Technologies (Euro 1000) and was invited as speaker at the International Conference on Cellvizio ${ }^{\circledR}$ Users in 2010 and 2011 (no fee) by Mauna Kea Technologies. MS was invited to attend the International Conference on Cellvizio ${ }^{\circledR}$ Users by Mauna Kea Technologies in 2011. TH, NP, SL declare no conflict of interest. None of the authors has a financial relationship with a commercial entity that has an interest with the subject of this manuscript. The authors disclose that the first case discussed in this series has been presented at the Chest Medicine Conference: The Art of Minimally Invasive Thoracic Surgery and Interventional Pulmonary Medicine: A Collaborative Approach, March 21-22, 2014, New York, USA.

Open Access This article is distributed under the terms of the Creative Commons Attribution 4.0 International License (http://crea tivecommons.org/licenses/by/4.0/), which permits unrestricted use, distribution, and reproduction in any medium, provided you give appropriate credit to the original author(s) and the source, provide a link to the Creative Commons license, and indicate if changes were made.

\section{References}

1. Pelosi G, Sonzogni A, Veronesi G et al (2008) Pathologic and molecular features of screening low-dose computed tomography (LDCT)-detected lung cancer: a baseline and 2-year repeat study. Lung Cancer 62:202-214

2. MacMahon H, Austin JH (2005) Guidleines for management of small pulmonary nodules detected on CT scans: a statement from the Fleischner Society. Radiology 237:395

3. Naidich DP, Bankier AA, MacMahon H (2013) Recommendations for the management of subsolid pulmonary nodules detected at CT: a statement from the Fleischner Society. Radiology 266:304
4. Oki M, Saka H, Kitagawa C et al (2012) Randomized study of endobronchial ultrasound-guided transbronchial biopsy: thin bronchoscopic method versus guide sheath method. J Thorac Oncol 7:535-541

5. Eberhardt R, Anantham D, Ernst A et al (2007) Multimodality bronchoscopic diagnosis of peripheral lung lesions: a randomized controlled trial. Am J Respir Crit Care Med 176:36-41

6. Wang Memoli JS, Nietert PJ, Silvestri GA (2012) Meta-analysis of guided bronchoscopy for the evaluation of the pulmonary nodule. Chest 142:385

7. Thiberville L, Moreno-Swirc S, Vercauteren T et al (2007) In vivo imaging of the bronchial wall microstructure using fibered confcal fluorescence microscopy. AJRCCM 175:22

8. Thiberville L, Salaun M, Lachkar S et al (2009) Confocal fluorescence endomicroscopy of the human airways. Proc Am Thorac Soc 6:444

9. Thiberville L, Salaun M, Lachkar S et al (2009) Human in vivo fluorescence microimaging of the alveolar ducts and sacs during bronchoscopy. Eur Respir J 33:974

10. Thiberville L, Salaun M (2010) Bronchoscopic advances: on the way to the cells. Respiratory 79(6):441

11. Asano F, Matsuno Y, Tsuzuku A et al (2008) Diagnosis of peripheral pulmonary lesions using a bronchoscope insertion guidance system combined with endobronchial ultrasonography with a guide sheath. Lung Cancer 60:366-373

12. Meredith P, Sarna T (2006) The physical and chemical properties of eumelanin. Pigment Cell Res 19(6):572-594

13. Gould MK, Donington J, Lynch WR et al (2013) Evaluation of individuals with pulmonary nodules: when is it lung cancer? Diagnosis and management of lung cancer, 3rd ed: American College of Chest Physicians evidence-based clinical practice guidelines. Chest 143(5 Suppl):e93S-e120S

14. McWilliams A, Tammemagi MC, Mayo JR, Roberts H, Liu G, Soghrati K, Yasufuku K, Martel S, Laberge F, Gingras M et al (2013) Probability of cancer in pulmonary nodules detected on first screening CT. N Eng J Med 369:910-919

15. Thiberville L, Salaun M, Lachkar S et al (2008) In-vivo confocal endomicroscopy of peripheral lung nodules using $488 \mathrm{~nm} /$ $660 \mathrm{~nm}$ induced fluorescence and topical methylene blue. Eur Respir J 32(Suppl):263s 